\title{
COMBINING ABILITY OF NEW YELLOW MAIZE INBRED LINES USING LINE X TESTER MATING DESIGN
}

\author{
Khalil, M.A.G.; S.M. Abo El-Hares; M.A.A. Hassan and E.A. \\ Amer \\ Maize Research Section, FCRI, ARC, Egypt
}

\begin{abstract}
General (GCA) and specific (SCA) combining ability were estimated in a lines $x$ testers crossing program comprising 36 crosses of maize produced by crossing eighteen yellow inbred lines with two testers in 2014 season. The resulting 36 test crosses along with the two check hybrids SC.162 and TWC353 were evaluated for six traits, i.e. days to 50\% silking, plant height, ear height, ear length, ear diameter and grain yield/ha at Sakha and Ismailia Agric. Res. Stations in 2015 grown season. Significant differences were obtained among crosses and their partitioning into lines, testers and line $x$ testers for all studied traits, except testers for ear diameter and line $\mathrm{x}$ tester for number of days to $50 \%$ silking, plant height, ear length, ear diameter and grain yield. The additive gene action was more important than non-additive gene action for days to $50 \%$ silking and grain yield, while the non-additive gene action was more important for other traits. The best inbred lines for GCA effects were Sk-5001/32 and Sk-5001/33 for days to 50\% silking, Sk-5001/36 and Sk-5001/38 for plant and ear height, Sk-5001/21, Sk-5001/29 and Sk$5001 / 36$ for ear length, Sk-5001/24, Sk-5001/29 and Sk-5001/36for ear diameter and Sk-5001/29, Sk-5001/30 and Sk-5001/36 for grain yield. Whereas, the line GZ-666 was the best tester for GCA effects for number of days to $50 \%$ silking and plant and ear height, while the single cross 162 was the best tester for ear length, ear diameter and grain yield. Two crosses (Sk$5001 / 25 \times$ GZ-666 and sk-5001/33 x SC 162) were found to be the best for SCA effects for grain yield. Most single crosses and all three way crosses were significantly outyielding both checks SC162 and TWC353, respectively.
\end{abstract}

Key words: Zea mays, GCA, SCA and Gene action.

\section{INTRODUCTION}

Maize (Zea mays L.) is one of the most important cereal crops in Egypt and the world. Maize in the world ranks the third surpassed only by wheat and rice. In Egypt, it is necessary to increase maize yield to face the wide gab between the production and consumption. High maize production can be achieved by improving cultural practices and planting the promising hybrids. In recent years, a major objective of the maize breeding program in Egypt is to develop high yielding yellow maize hybrids. The concept of combining ability, as a measure of gene action was proposed by Sprague and Tatum (1942). Combining ability 
and genetic variance components are important in the breeding programs for hybridization (Fehr, 1993). In any breeding program, the choice of the correct parents is the secret of the success. One of the most important criteria in breeding programs for identifying hybrids with high yield is knowledge regarding parent's genetic structure and information regarding their combining ability (Ceyhan 2003). Genetic information was obtained by different quantitative genetic methods. Line $\mathrm{x}$ tester analysis is a suitable and efficient method with eligible speed (Singh and Chaudhary, 1985). The line $x$ tester analysis method has been widely used by plant breeders. This method was suggested by Kempthorne (1957) and is used to breed both self and cross pollinated plants, as well as identifing favorable parents, crosses and estimating their general and specific combining ability effects, respectively. The additive gene action was more important in the inheritance of number of days to $50 \%$ silking (Soliman and Sadik 1999 and El-Shenawy and Mosa 2005) and grain yield (Aly and Khalil 2013, Mostafa et al. 2014 and El-Gazzar 2015). While, the non-additive gene action seemed to have played an important role in the expression of plant height (Aly and Khalil 2013), ear height (El-Gazzar and Khalil 2012 and El-Gazzar2015), ear length (El-Shenaway2005) and ear diameter (Amer 2004).

The objective of this study was to estimate the general and specific combining ability effects for new yellow inbred lines and to identify the superior crosses to be used in maize breeding programs.

\section{MATERIALS AND METHODS}

The experimental material consisted of eighteen yellow inbred lines, which have been designated as Sk-5001/21, Sk-5001/22, Sk5001/23, Sk-5001/24, Sk-5001/25, Sk-5001/26, Sk-5001/27, Sk-5001/28, Sk-5001/29, Sk-5001/30, Sk-5001/31, Sk-5001/32, Sk-5001/33, Sk$5001 / 34$, Sk-5001/35, Sk-5001/36,Sk-5001/37 and Sk-5001/38 developed at Sakha Agriculture Research Station. These lines and the two testers (inbred line GZ - 666 and single cross162) were crossed in line $x$ tester design, in 2014 summer season. In 2015 summer season, resulting 36 crosses along with the two commercial check hybrids i.e. SC162 and TWC 353 were evaluated in a randomized complete blocks design (RCBD) with four replications at two locations, i.e. Sakha and Ismailia Agricultural Research Stations. The plot consisted of one row, $6 \mathrm{~m}$ long , $0.8 \mathrm{~m}$ width and space between hills at $0.25 \mathrm{~m}$ along the row. Two kernels / hill were planted, then later thinned to one plant per hill. All cultural practices for maize production were applied as recommended at the proper time. Data were taken for number of days to $50 \%$ silking, plant height $(\mathrm{cm})$, ear height $(\mathrm{cm})$, ear length $(\mathrm{cm})$, ear diameter $(\mathrm{cm})$ and grain yield (adjusted to $15.5 \%$ moisture content) per plot and 
converted to ton per hectare. After recording the observation for each character, the analysis of variance was carried out according to Steel and Torrie (1980) for combined data across locations. The mean squares from line $x$ tester design and the general combining ability (GCA) and specific combining ability (SCA) variances and effects were calculated according to the procedures developed by Kempthorne (1957) and adopted by Singh and Choudhry(1979).

\section{RESULTS AND DISCUSSION}

Combined analysis of variance of 38 hybrids for six traits of maize across the two locations is given in Table 1. The results showed that the differences between locations (loc) were highly significant for all the studied traits, except for plant height and grain yield.

Differences between hybrids $(\mathrm{H})$ were highly significant for the all studied traits. Also, hybrids $x$ locations mean squares were significant or highly significant for all studied traits, indicating that the hybrids presented differential performance in the testing locations.

Mean performance of 36 hybrids and two check hybrids (SC162 and TWC 353) for the six traits across the two locations is presented in Table 2. For days to $50 \%$ silking, the new crosses ranged from 61.00 days for (Sk-5001/32 x GZ-666) to 67.25 days for (Sk-5001/31 x SC 162), most crosses were significantly earlier than the check hybrids (SC162 and TWC 353), the best hybrids from them were Sk-5001/32 x GZ-666 and Sk-5001/33 x SC 162. Regarding to plant and ear height, the means performance for the new crosses ranged from $242.25 \mathrm{~cm}$ to $298.50 \mathrm{~cm}$ for plant height and from $110.75 \mathrm{~cm}$ to $149.50 \mathrm{~cm}$ for ear height for the crosses Sk-5001/38 x GZ-666 and Sk-5001/36 x SC 162, respectively. Most of crosses were shorter in plant height and lower in ear placement compared with the two checks. As for ear length, the mean performance for the new crosses ranged from $18.62 \mathrm{~cm}$ for Sk$5001 / 25$ x GZ-666 to 21.91 for Sk-5001/29 x GZ-666, most new threeway crosses had higher ear length than the check TWC 353. For ear diameter, the new crosses varied from $4.43 \mathrm{~cm}$ for Sk-5001/21 x GZ666 to $4.88 \mathrm{~cm}$ for Sk-5001/30 x GZ-666.

As for grain yield, mean performance ranged from 10.15 ton / ha for Sk-5001/26 x GZ-666 to 12.29 ton/ha for Sk-5001/31 x GZ-666. Also, the results showed that nine new single crosses and all new three-way crosses significantly outyielded the checks SC 162 and TWC 353, respectively. The best hybrids from them were the single crosses Sk5001/30 x GZ-666, Sk5001/31 x GZ-666, Sk5001/36 x GZ666 and the three-way crosses Sk- 5001/29 x SC-162, Sk- 5001/31 x SC 162; Sk-5001/33 x SC 162; these hybrids will be tested in yield trails for further evaluation. 
Table 1: Combined analysis of variance for six quantitative traits across two locations

\begin{tabular}{|c|c|c|c|c|c|c|c|}
\hline S.O.V & D.F. & $\begin{array}{c}\text { Days to } \\
50 \% \\
\text { silking }\end{array}$ & $\begin{array}{c}\text { Plant } \\
\text { height }\end{array}$ & $\begin{array}{c}\text { Ear } \\
\text { height }\end{array}$ & $\begin{array}{c}\text { Ear } \\
\text { length }\end{array}$ & $\begin{array}{c}\text { Ear } \\
\text { diameter }\end{array}$ & $\begin{array}{c}\text { Grain } \\
\text { yield }\end{array}$ \\
\hline Location & 1 & $8410^{\star *}$ & 175.52 & $23573.45^{\star *}$ & $325.77^{* *}$ & $9.95^{\star *}$ & 0.576 \\
\hline Reps/Loc & 6 & 11.74 & 346.98 & 218.24 & 2.80 & 0.295 & 3.15 \\
\hline $\begin{array}{c}\text { Hybrids } \\
\text { (H) }\end{array}$ & 37 & $27.46^{\star *}$ & $1021.13^{\star *}$ & $914.31^{\star *}$ & $8.31^{* *}$ & $0.090^{\star *}$ & $4.615^{\star *}$ \\
\hline HxLoc & 37 & $5.71^{\star *}$ & $220.43^{*}$ & $145.24^{*}$ & $1.31^{* *}$ & $0.044^{*}$ & $4.130^{\star *}$ \\
\hline Error & 222 & 2.27 & 140.99 & 98.97 & 0.752 & 0.0276 & 0.925 \\
\hline CV\% & & 2.34 & 4.31 & 7.39 & 4.29 & 3.59 & 8.64 \\
\hline
\end{tabular}

${ }^{*}$ and ${ }^{* *}$ significant differences at 0.05 and 0.01 levels of probability, respectively.

Mean squares of line $x$ tester analysis for 36 crosses for eight traits across two locations are presented in Table 3 . The mean squares due to lines $(\mathrm{L})$ and testers $(T)$ were highly significant for all studied traits, except $(T)$ for ear diameter, indicating that the inbred lines behaved differently in their respective top crosses, and that greater diversity existed between testers. While, the $L \times T$ interaction was significant for plant height only, indicating that the inbred lines performed differently in their respective top crosses depending on the type of testers used for this trait. These results are in agreement with those obtained by Dodiya and Joshi (2002), Habliza and Khalifa (2005), Mosa(2010), El-Gazzar and Khalil (2012), Mostafa et al. (2014) and Motawi et al. (2016).

The mean squares due to interaction of locations with lines $(L)$, testers $(T)$ and their interactions $(L \times T)$ were not significant for all traits, except $L x$ loc for ear length and grain yield, $T \times$ Loc for days to $50 \%$ silking and $L \times T \times$ Loc for grain yield. These results are in accordance with those reported by Amer (2004), Mosa (2005), El-Gazzar et al. (2013), El-Gazzar (2015) and Motawei et al. (2016). 
Table (2): Mean performance of 36 crosses and two checks for six traits across two locations

\begin{tabular}{|c|c|c|c|c|c|c|}
\hline Cross & $\begin{array}{c}\text { Days to } 50 \% \\
\text { silking } \\
\text { (day) }\end{array}$ & $\begin{array}{l}\text { Plant height } \\
\text { (cm) }\end{array}$ & $\begin{array}{l}\text { Ear height } \\
\text { (cm) }\end{array}$ & $\begin{array}{l}\text { Ear length } \\
\text { (cm) }\end{array}$ & $\begin{array}{l}\text { Ear diameter } \\
\text { (cm) }\end{array}$ & $\begin{array}{l}\text { Grain yield } \\
\text { (ton/ha) }\end{array}$ \\
\hline Sk-5001/21 x GZ-666 & 63.62 & 254.37 & 118.75 & 20.22 & 4.43 & 11.45 \\
\hline Sk-5001/22 x GZ-666 & 61.87 & 267.62 & 121.00 & 19.62 & 4.61 & 10.78 \\
\hline Sk-5001/23 x GZ-666 & 64.00 & 278.12 & 130.37 & 18.76 & 4.51 & 10.69 \\
\hline Sk-5001/24 x GZ-666 & 62.75 & 290.25 & 134.87 & 19.13 & 4.81 & 10.91 \\
\hline Sk-5001/25 x GZ-666 & 63.62 & 273.50 & 127.50 & 18.62 & 4.61 & 11.28 \\
\hline Sk-5001/26 x GZ-666 & 62.25 & 264.87 & 119.37 & 19.76 & 4.57 & 10.15 \\
\hline Sk-5001/27 x GZ-666 & 62.50 & 274.75 & 124.50 & 20.20 & 4.57 & 10.74 \\
\hline Sk-5001/28 x GZ-666 & 63.12 & 269.87 & 141.00 & 20.20 & 4.51 & 11.01 \\
\hline Sk-5001/29 x GZ-666 & 63.25 & 284.37 & 129.25 & 21.91 & 4.77 & 11.08 \\
\hline Sk-5001/30 x GZ-666 & 63.87 & 265.00 & 126.62 & 18.85 & 4.88 & 12.00 \\
\hline Sk-5001/31 x GZ-666 & 63.87 & 278.62 & 128.25 & 19.68 & 4.70 & 12.29 \\
\hline Sk-5001/32 x GZ-666 & 61.00 & 271.25 & 125.25 & 19.46 & 4.62 & 10.20 \\
\hline Sk-5001/33 x GZ-666 & 61.62 & 267.00 & 112.75 & 19.73 & 4.66 & 10.51 \\
\hline Sk-5001/34 x GZ-666 & 62.50 & 280.62 & 132.37 & 19.75 & 4.55 & 10.43 \\
\hline Sk-5001/35 x GZ-666 & 62.25 & 278.87 & 139.50 & 19.78 & 4.40 & 11.04 \\
\hline Sk-5001/36 x GZ-666 & 62.25 & 260.75 & 113.37 & 20.76 & 4.47 & 12.04 \\
\hline Sk-5001/37 x GZ-666 & 63.25 & 265.12 & 116.75 & 19.33 & 4.50 & 11.40 \\
\hline Sk-5001/38 x GZ-666 & 62.25 & 242.25 & 110.75 & 19.30 & 4.66 & 10.43 \\
\hline Sk-5001/21 x SC 162 & 66.00 & 276.50 & 139.50 & 21.65 & 4.62 & 11.86 \\
\hline Sk-5001/22 x SC 162 & 64.25 & 284.25 & 138.62 & 20.50 & 4.58 & 11.58 \\
\hline Sk-5001/23 x SC 162 & 65.62 & 275.25 & 136.12 & 20.03 & 4.53 & 10.94 \\
\hline Sk-5001/24 x SC 162 & 66.00 & 291.37 & 142.50 & 19.58 & 4.73 & 11.73 \\
\hline Sk-5001/25 x SC 162 & 66.87 & 280.37 & 133.50 & 19.28 & 4.52 & 10.34 \\
\hline Sk-5001/26 x SC 162 & 65.75 & 277.00 & 132.50 & 20.31 & 4.65 & 10.16 \\
\hline Sk-5001/27 x SC 162 & 64.87 & 279.12 & 138.12 & 20.85 & 4.57 & 11.27 \\
\hline Sk-5001/28 x SC 162 & 66.62 & 271.87 & 140.50 & 21.03 & 4.62 & 11.73 \\
\hline Sk-5001/29 x SC 162 & 66.12 & 286.50 & 142.12 & 21.07 & 4.68 & 12.20 \\
\hline Sk-5001/30 x SC 162 & 67.12 & 275.62 & 140.12 & 19.87 & 4.67 & 11.77 \\
\hline Sk-5001/31 x SC 162 & 67.25 & 283.50 & 145.00 & 20.58 & 4.68 & 12.04 \\
\hline Sk-5001/32 x SC 162 & 64.75 & 270.50 & 139.25 & 20.01 & 4.60 & 11.17 \\
\hline Sk-5001/33 x SC 162 & 64.00 & 281.37 & 143.50 & 20.81 & 4.65 & 11.94 \\
\hline Sk-5001/34 x SC 162 & 65.75 & 289.75 & 148.50 & 20.48 & 4.75 & 10.94 \\
\hline Sk-5001/35 x SC 162 & 66.62 & 298.50 & 149.50 & 20.90 & 4.55 & 10.23 \\
\hline Sk-5001/36 x SC 162 & 64.25 & 266.50 & 132.25 & 21.36 & 4.72 & 11.79 \\
\hline Sk-5001/37 x SC 162 & 65.37 & 279.25 & 136.00 & 20.36 & 4.56 & 11.35 \\
\hline Sk-5001/38 x SC 162 & 64.62 & 255.75 & 120.37 & 21.48 & 4.68 & 11.77 \\
\hline SC 162 & 68.00 & 291.12 & 144.37 & 23.78 & 4.70 & 9.97 \\
\hline TWC353 & 67.12 & 280.00 & 150.00 & 18.87 & 4.80 & 8.91 \\
\hline LSD at 0.05 & 1.47 & 11.63 & 9.75 & 0.84 & 0.16 & 0.94 \\
\hline
\end{tabular}

Table 3: Line $x$ tester analysis of 36 top crosses for six traits combined across two locations

\begin{tabular}{|c|c|c|c|c|c|c|c|}
\hline S.O.V & D.F & $\begin{array}{l}\text { Days to } \\
50 \% \\
\text { silking }\end{array}$ & $\begin{array}{l}\text { Plant } \\
\text { height }\end{array}$ & Ear height & $\begin{array}{l}\text { Ear } \\
\text { length }\end{array}$ & $\begin{array}{l}\text { Ear } \\
\text { diameter }\end{array}$ & $\begin{array}{l}\text { Grain yield } \\
\text { (ton/hectar) }\end{array}$ \\
\hline Lines (L) & 17 & $12.19^{\star *}$ & $1576.17^{\star \star}$ & $756.52^{\star \star}$ & $6.81^{\star \star}$ & $0.12^{\star \star}$ & $4.37^{\star \star}$ \\
\hline Testers(T) & 1 & $600.88^{\star *}$ & $5390.68^{\star *}$ & $13708.92^{\star *}$ & $50.66^{\star *}$ & 0.06 & $10.34^{\star *}$ \\
\hline $\mathrm{L} \times \mathrm{T}$ & 17 & 2.05 & 206.30 & $244.30^{*}$ & 1.36 & 0.05 & 1.98 \\
\hline L X Loc & 17 & 3.258 & 137.728 & 125.928 & $1.185^{*}$ & 0.043 & $3.604^{* *}$ \\
\hline $\mathrm{T} \times \mathrm{LOC}$ & 1 & $55.125^{\star *}$ & 43.556 & 21.67 & 2.801 & 0.056 & 0.212 \\
\hline Lx T x Loc & 17 & 2.324 & 118.857 & 147.509 & 1.024 & 0.043 & $3.321^{\star *}$ \\
\hline Error & 222 & 2.27 & 140.99 & 98.97 & 0.752 & 0.0276 & 0.925 \\
\hline
\end{tabular}

* and ${ }^{* *}$ significant differences at 0.05 and 0.01 levels of probability, respectively.

Additive and non-additive genetic variances and their interaction with locations are presented in Table (4). The additive genetic effects $\left(\sigma^{2} \mathrm{GCA}\right)$ seemed to have played more important role than non-additive genetic effects $\left(\sigma^{2} S C A\right)$ in the inheritance of number days to $50 \%$ silking and grain yield, while, non-additive gene action ( $\left.\sigma^{2} \mathrm{SCA}\right)$ was 
effective and played an important role in the inhertance of plant height, ear height, ear length and ear diameter. These results support the findings of Amer (2004), Motawei and Mosa (2009) and Mostafa et al. (2014) for number days to 50\% silking, Mosa (2004), Motawei (2006) and Aly and Khalil (2013) for plant height, Amer (2004) El-Gazzar and Khalil (2012) and El-Gazzar (2015) for ear height, Mosa (2004) and ElShenaway (2005) for ear length, Amer (2004) for ear dimeter and Aly and Khalil (2013), Mostafa et al. (2013) and El-Gazzar (2015) for grain yield.

Table 4: Genetic components for six quantitative traits across two locations

\begin{tabular}{|l|l|l|l|l|l|l|}
\hline $\begin{array}{l}\text { Variance } \\
\text { components }\end{array}$ & $\begin{array}{l}\text { Days to } \\
50 \% \text { silking }\end{array}$ & $\begin{array}{l}\text { Plant } \\
\text { height }\end{array}$ & $\begin{array}{l}\text { Ear } \\
\text { height }\end{array}$ & $\begin{array}{l}\text { Ear } \\
\text { length }\end{array}$ & $\begin{array}{l}\text { Ear } \\
\text { diameter }\end{array}$ & $\begin{array}{l}\text { Grain yield } \\
\text { (ton/ha) }\end{array}$ \\
\hline$\sigma^{2}$ GCA & 0.217 & 6.351 & 6.723 & 0.034 & 0.0002 & 0.006 \\
\hline$\sigma^{2}$ SCA & 0.0001 & 10.93 & 12.090 & 0.042 & 0.001 & 0.0001 \\
\hline
\end{tabular}

Estimates of general combining ability effects of eighteen inbred lines and two testers for the six traits across the two locations are presented in Table 5. The excellent inbred lines for general combining ability effects were Sk-5001/22, Sk- 5001/32, Sk- 5001/33, Sk-5001/36 and Sk-5001/38for earliness; Sk- 5001/36 and Sk- 5001/38 for short plant and ear height; Sk-5001/21, Sk-5001/29 and Sk-5001/36 for ear length, Sk-5001/24, Sk-5001/29 and Sk- 5001/30 for ear diameter and Sk-5001/29, Sk-5001/30, Sk-5001/31 and Sk-5001/36 for grain yield. In general, the best line for general combining ability was Sk-5001/36 for most studied traits.

Table 5: Estimates of general combining ability $\left(\mathrm{gi}_{\mathrm{i}}\right)$ effects of 18 inbred lines and 2 testers for six traits across two locations

\begin{tabular}{|c|c|c|c|c|c|c|c|}
\hline \multicolumn{2}{|c|}{ Inbred line/ Testers } & $\begin{array}{l}\text { Days to } 50 \% \\
\text { silking }\end{array}$ & $\begin{array}{l}\text { Plant } \\
\text { height }\end{array}$ & $\begin{array}{c}\text { Ear } \\
\text { height }\end{array}$ & $\begin{array}{l}\text { Ear } \\
\text { length }\end{array}$ & Ear diameter & $\begin{array}{l}\text { Grain } \\
\text { yield }\end{array}$ \\
\hline \multicolumn{2}{|l|}{ Sk-5001/21 } & 0.597 & $-9.291^{\star \star}$ & -2.899 & $0.788^{\star \star}$ & $-0.088^{\star}$ & 0.441 \\
\hline \multicolumn{2}{|l|}{ Sk-5001/22 } & $-1.152^{\star \star}$ & 1.208 & -2.211 & -0.086 & -0.020 & -0.032 \\
\hline \multicolumn{2}{|l|}{ Sk-5001/23 } & 0.597 & 1.958 & 1.225 & $-0.748^{\star \star}$ & $-0.095^{*}$ & -0.397 \\
\hline \multicolumn{2}{|l|}{ Sk-5001/24 } & 0.159 & $16.083^{\star \star \star}$ & $6.663^{\star \star}$ & $-0.786^{\star \star}$ & $0.154^{\star \star}$ & 0.104 \\
\hline \multicolumn{2}{|l|}{ Sk-5001/25 } & $1.034^{\star \star}$ & 2.208 & -1.524 & $-1.192^{\star \star}$ & -0.051 & -0.406 \\
\hline \multicolumn{2}{|l|}{ Sk-5001/26 } & -0.215 & -3.791 & $-6.086^{\star}$ & -0.111 & -0.007 & $-1.062^{\star \star}$ \\
\hline \multicolumn{2}{|l|}{ Sk-5001/27 } & -0.527 & 2.208 & -0.711 & 0.376 & -0.045 & 0.017 \\
\hline \multicolumn{2}{|l|}{ Sk-5001/28 } & 0.659 & -3.854 & $8.725^{\star \star}$ & $0.470^{\star}$ & -0.051 & 0.155 \\
\hline \multicolumn{2}{|l|}{ Sk-5001/29 } & 0.472 & $10.708^{* \star}$ & 3.663 & $1.345^{\star \star}$ & $0.111^{\star \star}$ & $0.426^{*}$ \\
\hline \multicolumn{2}{|l|}{ Sk-5001/30 } & $1.284^{\star \star}$ & -4.416 & 1.350 & $-0.786^{\star \star}$ & $0.161^{\star \star}$ & $0.674^{\star \star}$ \\
\hline \multicolumn{2}{|l|}{ Sk-5001/31 } & $1.347^{\star \star}$ & $6.333^{\star}$ & 4.600 & -0.011 & 0.073 & $0.951^{\star \star}$ \\
\hline \multicolumn{2}{|l|}{ Sk-5001/32 } & $-1.340^{\star \star}$ & -3.854 & 0.225 & -0.411 & -0.007 & $-0.526^{\star}$ \\
\hline \multicolumn{2}{|l|}{ Sk-5001/33 } & $-1.402^{\star \star}$ & -0.541 & -3.586 & 0.126 & 0.036 & 0.010 \\
\hline \multicolumn{2}{|l|}{ Sk-5001/34 } & -0.090 & $10.458^{\star \star}$ & $8.413^{\star \star}$ & -0.029 & 0.029 & $-0.528^{\star}$ \\
\hline \multicolumn{2}{|l|}{ Sk-5001/35 } & 0.222 & $13.958^{\star \star}$ & $12.475^{\star \star}$ & 0.195 & $-0.145^{\star \star}$ & $-0.577^{\star}$ \\
\hline \multicolumn{2}{|l|}{ Sk-5001/36 } & $-0.965^{\star}$ & $-11.104^{\star \star}$ & $-9.416^{\star \star}$ & $0.913^{\star \star}$ & -0.020 & $0.700^{\star \star}$ \\
\hline \multicolumn{2}{|l|}{ Sk-5001/37 } & 0.097 & -2.541 & $-5.649^{\star}$ & -0.29 & $-0.088^{*}$ & 0.164 \\
\hline \multicolumn{2}{|l|}{ Sk-5001/38 } & $-0.777^{\star}$ & $-25.729^{\star \star}$ & $-15.211^{\star \star}$ & 0.245 & 0.054 & 0.115 \\
\hline \multirow{2}{*}{\multicolumn{2}{|c|}{$\begin{array}{l}\text { Tester GZ-666 } \\
\text { Tester SC-162 }\end{array}$}} & $-1.444^{\star \star}$ & $-4.326^{\star \star}$ & $-6.899^{\star \star}$ & $-0.419^{\star \star}$ & -0.015 & $-0.189^{\star}$ \\
\hline & & $1.444^{\star \star}$ & $4.326^{\star \star}$ & $6.899^{\star \star}$ & $0.419^{\star \star}$ & 0.015 & $0.189^{\star}$ \\
\hline \multirow[b]{2}{*}{ LSD lines $g_{i}$} & 0.05 & 0.738 & 5.81 & 4.87 & 0.42 & 0.081 & 0.47 \\
\hline & 0.01 & 0.971 & 7.65 & 6.41 & 0.55 & 0.107 & 0.62 \\
\hline \multirow{2}{*}{ LSD testers $\mathrm{g}_{\mathrm{I}}$} & 0.05 & 0.24 & 1.93 & 1.62 & 0.14 & 0.027 & 0.15 \\
\hline & 0.01 & 0.32 & 2.55 & 2.13 & 0.18 & 0.035 & 0.20 \\
\hline
\end{tabular}


Estimates of general combining ability effects of two testers for the six traits across the two locations are presented in Table 5. The best tester for general combining ability effects was the inbred line GZ666 for earliness and short plants and low ear placement and SC162 for ear length, ear diameter and grain yield. Estimates of specific combining ability effects of the 36 top crosses for the six traits across the two locations are presented in Table 6. The desirable SCA effects were obtained from the crosses Sk-5001/35 x GZ-666 for earliness ; Sk-5001/21 x GZ-666 for plant height; Sk-5001/33 x GZ-666 for ear height; Sk-5001/29 x GZ-666 for ear length ; Sk-5001/30 x GZ-666 ear diameter and Sk-5001/25 x GZ-666 and sk-5001/33 x SC 162 for grain yield.

Table 6: Estimates of specific combining ability effects of 36 crosses for eight traits across two locations

\begin{tabular}{|c|c|c|c|c|c|c|}
\hline Crosses & $\begin{array}{c}\text { Days to } \\
50 \% \\
\text { silking }\end{array}$ & $\begin{array}{l}\text { Plant } \\
\text { height }\end{array}$ & $\begin{array}{c}\text { Ear } \\
\text { height }\end{array}$ & $\begin{array}{l}\text { Ear } \\
\text { length }\end{array}$ & $\begin{array}{c}\text { Ear } \\
\text { diameter }\end{array}$ & $\begin{array}{l}\text { Grain } \\
\text { yield }\end{array}$ \\
\hline Sk-5001/21 x GZ-666 & 0.256 & -6.736 & -3.475 & -0.293 & -0.078 & -0.019 \\
\hline Sk-5001/21 x SC162 & -0.256 & 6.736 & 3.475 & 0.293 & 0.078 & 0.019 \\
\hline Sk-5001/22 x GZ-666 & 0.256 & -3.986 & -1.913 & -0.018 & 0.027 & -0.207 \\
\hline Sk-5001/22 x SC162 & -0.256 & 3.986 & 1.913 & 0.018 & -0.027 & 0.207 \\
\hline Sk-5001/23 x GZ-666 & 0.631 & 5.763 & 4.024 & -0.218 & 0.002 & 0.065 \\
\hline Sk-5001/23 x SC162 & -0.631 & -5.763 & -4.024 & 0.218 & -0.002 & -0.065 \\
\hline Sk-5001/24 x GZ-666 & -0.180 & 3.763 & 3.086 & 0.194 & 0.052 & -0.220 \\
\hline Sk-5001/24 x SC162 & 0.180 & -3.763 & -3.086 & -0.194 & -0.052 & 0.220 \\
\hline Sk-5001/25 x GZ-666 & -0.180 & 0.888 & 3.899 & 0.088 & 0.059 & $0.660^{*}$ \\
\hline Sk-5001/25 x SC162 & 0.180 & -0.888 & -3.899 & -0.088 & -0.059 & $-0.660^{*}$ \\
\hline Sk-5001/26 x GZ-666 & -0.305 & -1.736 & 0.336 & 0.144 & -0.022 & 0.183 \\
\hline Sk-5001/26 x SC162 & 0.305 & 1.736 & -0.336 & -0.144 & 0.022 & -0.183 \\
\hline Sk-5001/27 x GZ-666 & 0.256 & 2.138 & 0.086 & 0.094 & 0.015 & -0.302 \\
\hline Sk-5001/27 x SC162 & -0.256 & -2.138 & -0.086 & -0.094 & -0.015 & 0.302 \\
\hline Sk-5001/28 x GZ-666 & -0.305 & 3.326 & $7.149^{*}$ & 0.001 & -0.040 & -0.166 \\
\hline Sk-5001/28 x SC162 & 0.305 & -3.326 & $-7.149^{*}$ & -0.001 & 0.040 & 0.166 \\
\hline Sk-5001/29 x GZ-666 & 0.006 & 3.263 & 0.461 & $0.838^{* *}$ & 0.059 & -0.367 \\
\hline Sk-5001/29 x SC162 & -0.006 & -3.263 & -0.461 & $-0.838^{* *}$ & -0.059 & 0.367 \\
\hline Sk-5001/30 x GZ-666 & -0.180 & -0.986 & 0.149 & -0.093 & $0.121^{*}$ & 0.303 \\
\hline Sk-5001/30 x SC162 & 0.180 & 0.986 & -0.149 & 0.093 & $-0.121^{*}$ & -0.303 \\
\hline Sk-5001/31 x GZ-666 & -0.243 & 1.888 & -1.457 & -0.030 & 0.021 & 0.310 \\
\hline Sk-5001/31 x SC162 & 0.243 & -1.888 & 1.457 & 0.030 & -0.021 & -0.310 \\
\hline Sk-5001/32 x GZ-666 & -0.430 & 4.701 & -0.100 & 0.144 & 0.027 & -0.297 \\
\hline Sk-5001/32 x SC162 & 0.430 & -4.701 & 0.100 & -0.144 & -0.027 & 0.297 \\
\hline Sk-5001/33 x GZ-666 & 0.256 & -2.861 & $-10.788^{\star \star}$ & -0.118 & 0.021 & -0.526 \\
\hline Sk-5001/33 x SC162 & -0.256 & 2.861 & $10.788^{\star \star}$ & 0.118 & -0.021 & 0.526 \\
\hline Sk-5001/34 x GZ-666 & -0.180 & -0.236 & -1.163 & 0.050 & -0.084 & -0.061 \\
\hline Sk-5001/34 x SC162 & 0.180 & 0.236 & 1.163 & -0.050 & 0.084 & 0.061 \\
\hline Sk-5001/35 x GZ-666 & -0.743 & -5.486 & 1.899 & -0.136 & -0.059 & 0.596 \\
\hline Sk-5001/35 x SC162 & 0.743 & 5.486 & -1.899 & 0.136 & 0.059 & -0.596 \\
\hline Sk-5001/36 x GZ-666 & 0.444 & 1.451 & 3.461 & 0.119 & -0.109 & 0.317 \\
\hline Sk-5001/36 x SC162 & -0.444 & -1.451 & -3.461 & -0.119 & 0.109 & -0.317 \\
\hline Sk-5001/37 x GZ-666 & 0.381 & -2.736 & -2.725 & -0.093 & -0.015 & 0.215 \\
\hline Sk-5001/37 x SC162 & -0.381 & 2.736 & 2.725 & 0.093 & 0.015 & -0.215 \\
\hline Sk-5001/38 x GZ-666 & 0.256 & -2.423 & -2.913 & $-0.674^{*}$ & 0.002 & -0.481 \\
\hline Sk-5001/38 x SC162 & -0.256 & 2.423 & 2.913 & $0.674^{*}$ & -0.002 & 0.481 \\
\hline LSD $_{\mathrm{ij}} 0.05$ & 1.04 & 8.22 & 6.89 & 0.60 & 0.11 & 0.66 \\
\hline LSD S $_{\mathrm{ij}} 0.01$ & 1.37 & 10.82 & 9.07 & 0.79 & 0.15 & 0.87 \\
\hline
\end{tabular}

${ }^{*}$ and ${ }^{* *}$ significant at 0.05 and 0.01 levels of probability, respectively. 


\section{REFERENCES}

Aly, R.S.H. and M.A.G. Khalil (2013). Combining ability for sixteen promising white maize inbred lines for grain yield and yield component traits. Egypt. J. Plant Breed. 17(3):143-160.

Amer, E.A. (2004). Combining ability of new white inbred lines of maize with three testers tested over two locations. Annals of Agric. Sc. Moshtohor, Vol. 42(2): 461-474.

Ceyhan, E. (2003). Determination of some agricultural characters and their heredity through line $x$ tester method in Pea parents and crosses. Selcuk Univ., Graduate School Nat. Applied Sci., Pp.130.

Dodiya , N. S. and V. N. Joshi (2002).Gene action for grain yield and its attributes in maize (Zea mays L.). Indian J. of Gent. and Plant Breed., 62(3):253-254.

El-Gazzar, I.A.I. and M.A.G. Khalil (2012). Combining ability analysis of new yellow inbred lines using line $x$ tester analysis. J. Agric. Res. Kafr ElSheikh Univ.,38(3): 390-400.

EL- Gazzar, I. A. I. ; M. A .EL-Ghonemy and S.Th. Mousa (2013).

Evaluation of new inbred lines of white maize via line $x$ tester analysis over three locations. J. Plant Production, Mansoura Univ., Vol. 4 (6): 897 - 906.

El-Gazzar, I.A.I.(2015). Combining ability for growth, yield and yield its attributes and resistance to downy mildew disease in maize. J. Agric. Res. Kafr El-Sheikh Univ.,41(2): 477-487.

El-Shenawy, A.A. (2005). Estimation of genetic and environment parameters for white inbred lines of maize (Zea mays L.). J. Agric Res Tanta Univ., 31(A-4):647-662.

El-Shenawy, A.A. and H.E. Mosa (2005). Evaluation of new single and three way crosses for resistance to downy mildew disease and grain yield under different environments. Alex. J. Agric. Res. 50:35-43Hallauer, A. R. (1990). Methods used in developing inbred. Maydica ,35:1-6.

Fehr, W.R. (1993). Principles of Cultivar Development. MacMillan Publication Co. New York, Pp.342.

Habliza , A. A. and K. I. Khalifa (2005). Selection among new yellow maize inbred lines using top crosses and stability analysis. Alex. J. Agric. Res. $50 ; 41-51$.

Kempthorne, O. (1957). An Introduction to Genetic Statistics. John Wiley and Sons Inc., New York, U.S.A.

Mosa, H.E.(2004). Comparison between two types of testers for evaluation of new white inbred lines of maize. Annals of Agric. Sc., Moshtohor, Vol.42(2): 475-487.

Mosa, H. E. (2005). Combining ability of eight yellow maize ( Zea mays L.) inbred lines for different characters in diallel crosses. J. Agric Res. Tanta Univ., 31(4-A): 604-615. 
Mosa, H. E. (2010).Estimation of combining ability of maize inbred lines using top cross mating design. J. Agric. Res. Kafr El-Sheikh Univ.,36(1):1-16.

Mostafa, M.A.A., M.A.G. Khalil and El-Gazzar, I.A.I.(2013). Estimation of combining ability of new white maize inbred lines using line $x$ tester mating design. Egypt. J. Plant Breed. 17:(2), 297-305.

Motawei A.A. (2006). Additive and non-additive genetic variances of important quintative traits in new maize inbred lines via line $x$ tester analysis. J. Agric. Sci. Mansoura Univ., 31(11): 6855-6865.

Motawei, A.A. and H.E. Mosa (2009). Genetic analysis for some quantitative traits in yellow maize via half diallel design. Egypt. J. plant Breed. 13:223-233.

Motawei, A.A.; M.A.G. Khalil; M.A.A. Hassan and E.A. Amer (2016). Superiority over check variety and combining ability based on line $x$ tester analysis in maize. Egypt J. Plant Breed. 20(2):341-352.

Singh, R.K. and D.B. Chaudary (1979). Biometrical methods in quantitative genetic analysis. Kalyani Publisher, Baharate, Ram Road, Daryagani, New Delhi, India.

Singh, R.K. and D.B. Chaudhary (1985). Biometrical Methods in Quantitative Genetic Analysis. Kalyani Publisher, Baharate, Ram Road, Daryagani, New Delhi, India.

Sprague, G.F. and L.A. Tatum (1942). General vs. specific combining ability in single crosses of corn. J. Amer. Soc. Agron. 34: $923-$ 932.

Soliman, F. H. S. and S. E. Sadek (1999). Combining ability of new maize inbred lines and its utilization in the Egyptian hybrid program. Bull. Fac. Agric., Cairo Univ.50:1-20.

Steel, R. G. and J. H. Torrie (1980). Principals and Procedures of Statistics. Mc. Grow Hill Book Inc., New York, USA.

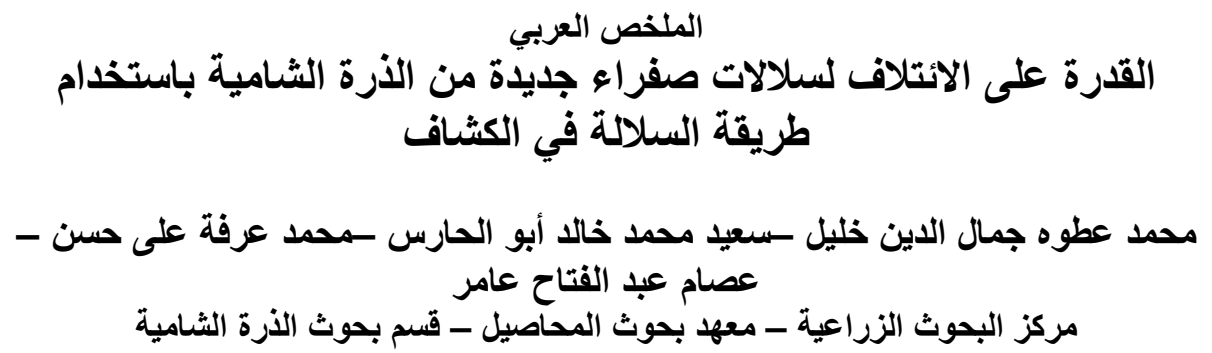

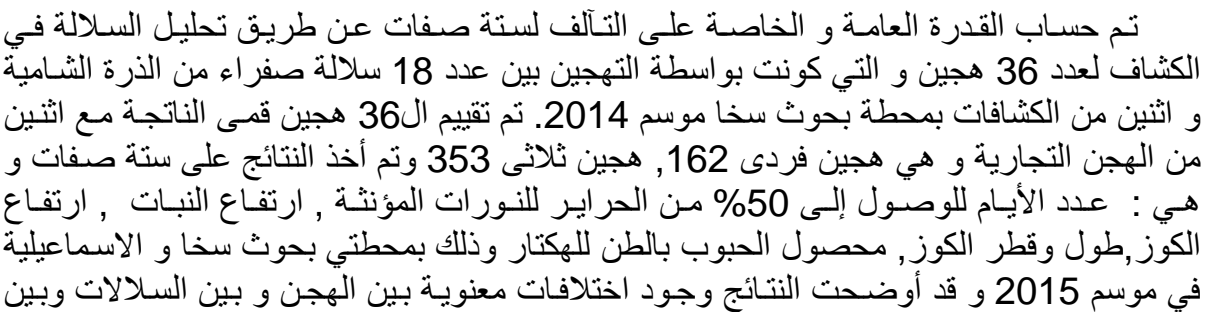




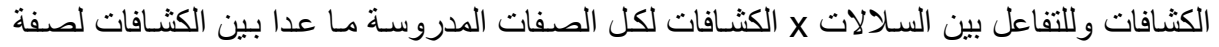

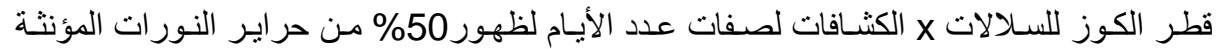

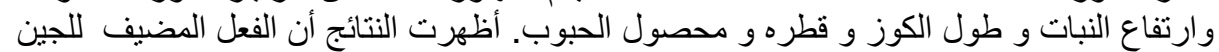

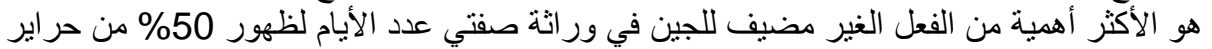

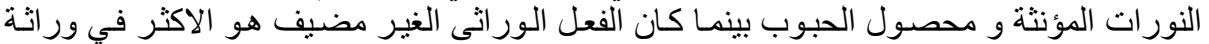

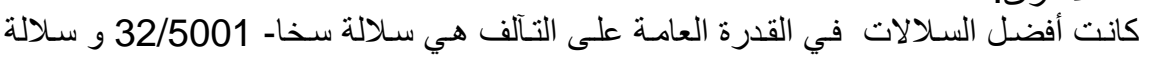

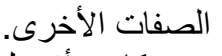

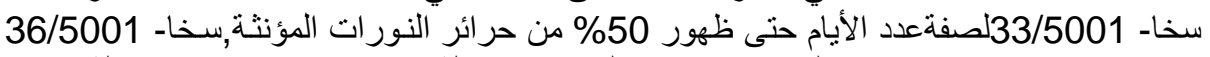

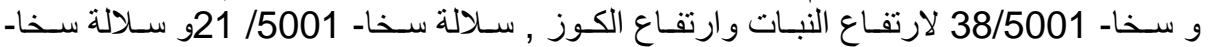

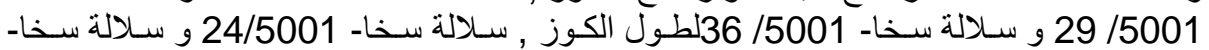

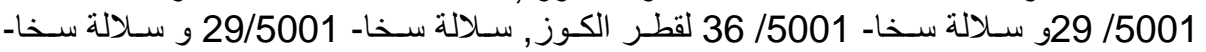

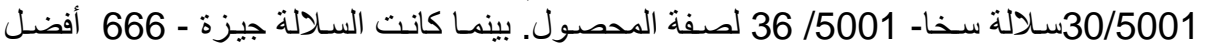

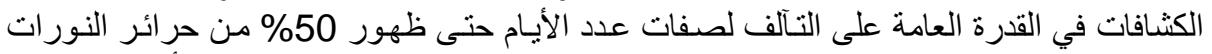

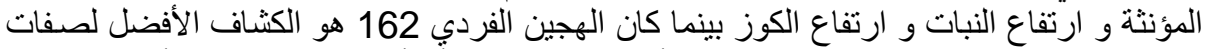

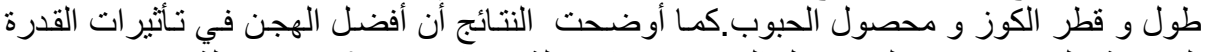

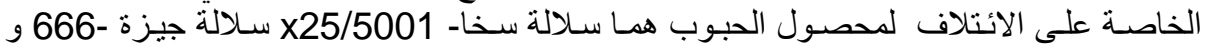

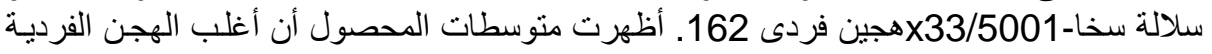

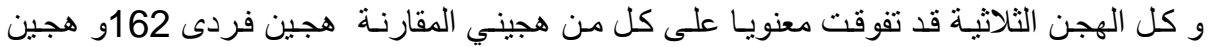

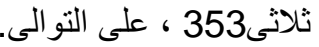

